\title{
miR-186 reverses cisplatin resistance and inhibits the formation of the glioblastoma-initiating cell phenotype by degrading Yin Yang 1 in glioblastoma
}

\author{
JIAN LI ${ }^{1}$, JIE SONG $^{2}$ and FENG GUO ${ }^{1}$ \\ ${ }^{1}$ Department of Neurosurgery, Linyi People's Hospital, Linyi, Shandong 276003; \\ ${ }^{2}$ Department of Neurosurgery, Yishui Central Hospital, Yishui, Shandong 276400, P.R. China
}

Received June 16, 2016; Accepted October 8, 2018

DOI: $10.3892 /$ ijmm.2018.3940

\begin{abstract}
Glioblastoma multiforme (GBM) is among the most devastating types of cancer, with a median survival of $<1$ year. Despite the development of new surgical and radiation techniques, and the use of multiple anti-neoplastic drugs, effective treatment strategies for malignant gliomas have not yet been developed. The limited efficacy of current treatments reflects the resistance of glioblastoma cells to cytotoxic agents. In this study, using western blot analysis, we found that Yin Yang 1 (YY1) expression was increased in cisplatin-resistant glioblastoma U87MG cells (U87MG-CR). We observed that the silencing of YY1 sensitized the U87MG-CR cells to cisplatin and that the overexpression of YY1 promoted the resistance of LN-229 glioblastoma cells to cisplatin, as shown by MTT assay. Using sphere formation assay, we also found that the silencing of YY1 inhibited the formation of the glioblastoma-initiating cell (GIC) phenotype in the U87MG-CR cells. In addition, the results of RT-qPCR revealed that miR-186 expression was decreased in U87MG-CR cells. Using RT-PCR and western blot analysis, we observed that overexpression of miR-186 inhibited YY1 expression in U87MG-CR cells. The overexpression of miR-186 also reversed cisplatin resistance and the formation of the GIC phenotype in glioblastoma cells. On the whole, the findings of this study demonstrate that miR-186 reverses cisplatin resistance and inhibits the formation of the GIC phenotype by degrading YY1 in glioblastoma.
\end{abstract}

\section{Introduction}

Glioblastoma is the most frequent primary malignant brain tumor among adults. The median survival is generally $<1$ year

Correspondence to: Dr Feng Guo, Department of Neurosurgery, Linyi People's Hospital, 27 Jiefang Road, Lanshan, Linyi, Shandong 276003, P.R. China

E-mail: guofeng00sd@163.com

Key words: glioblastoma, cisplatin resistance, glioblastoma-initiating cells, Yin Yang 1, microRNA-186 from the time of diagnosis, and even in the most favorable situations, the majority of patients succumb to the disease within 2 years (1-3). Standard therapy consists of surgical resection if that is safely feasible, followed by radiotherapy. However, the 5-year survival rate is $<3 \%$ (4). One of the reasons for the dismal prognosis is that current treatment strategies cannot eliminate glioblastoma-initiating cells (GICs) (5-7). A comprehensive understanding of the molecular basis of GICs may contribute to the identification of novel therapeutic targets.

Yin Yang 1 (YY1) is an ubiquitously expressed zinc finger transcription factor encoded by the $23 \mathrm{~kb}$ YY1 gene (8-12). Comprised of 414 amino acids, YY1 carries out various cellular functions, including transcriptional regulation, cell proliferation, chromatin remodeling and apoptosis (12-16). YY1 regulates multiple targets, including Erb-B2 receptor tyrosine kinase 2 (ERBB2), p53, caspases and histone deacetylases (HDACs), which have been implicated in cancer progression (15). YY1 expression has been shown to be increased in many types of cancer, including metastatic breast cancer $(17,18)$, colon cancer (19), gastric cancer (20) and prostate cancer (21). However, its roles have not yet been fully elucidated as regards the formation of GICs.

MicroRNAs (miRNAs or miRs), which are single-stranded long non-coding RNAs of 19-25 nucleotides in length, play important roles in the regulation of drug resistance and GICs $(22,23)$. miR-186 has been demonstrated to play a significant role as a tumor suppressor in many types of cancer (24-26). For example, miR-186 is a novel tumor suppressor miRNA that functions to inhibit tumorigenesis in glioblastoma multiforme (GBM) both in vitro and in vivo, by targeting both FGF2 and RelA (27); miR-186 may be a molecular target of glioblastoma (27). However, the role of miR-186 in GIC and drug resistance remains elusive. In this study, we observed that miR-186 reversed cisplatin resistance and inhibited the formation of the GIC phenotype by degrading YY1 in glioblastoma.

\section{Materials and methods}

Human glioblastoma cell lines. U87MG cells (glioblastoma of unknown origin) and LN-229 glioblastoma cells were purchased from then Biochemistry and Cell Biology Institute of Shanghai, Chinese Academy of Sciences (Shanghai, China), 
within 3 months of the experiments. Of note, it has been reported that the U87MG cell line has been misidentified (28). The U87 cell line used has been authenticated by STR profiling; thus, misidentification is not likely to affect the outcomes of this study. To obtain cisplatin-resistant glioblastoma U87MG cells (U87MG-CR cells), the U87MG cells were treated with escalating concentrations of cisplatin from $10^{7}$ to $10^{5} \mathrm{M}$ as previously reported (29). The established U87MG-CR cells grew at a similar rate in the presence or absence of $10^{5} \mathrm{M}$ cisplatin for 3 days (data not shown). The half maximal inhibitory concentration $\left(\mathrm{IC}_{50}\right)$ of the U87MG-CR cells increased by 12-fold, as compared with that of the U87MG cells (data not shown). The cells were cultured in Dulbecco's modified Eagle's medium (DMEM; Invitrogen, Shanghai, China) supplemented with 10\% fetal bovine serum (FBS; Invitrogen) and antibiotics $(100 \mathrm{mg} / \mathrm{ml}$ penicillin/100 U/ml streptomycin (Invitrogen) in a $5 \% \mathrm{CO}_{2}$ incubator at $37^{\circ} \mathrm{C}$.

shYY1 plasmids and pre-miR-186 and control miR. The shYY1 plasmids and scramble control were purchased from Tiangen (Beijing, China). Pre-miR-186 and control miR were purchased from Ambion, Inc. (Ambion, Austin, TX, USA).

Transfection experiment. Cell transfection was performed as previously described (30). For the transfection experiments, the cells were cultured in serum-free medium without antibiotics at $60 \%$ confluence for $24 \mathrm{~h}$, and then transfected using FuGENE HD transfection reagent (Roche, Indianapolis, IN, USA) according to the manufacturer's instructions. Following incubation for $6 \mathrm{~h}$ in a $5 \% \mathrm{CO}_{2}$ incubator at $37^{\circ} \mathrm{C}$, the medium was removed and replaced with normal culture medium (serum-free medium without antibiotics) for $24 \mathrm{~h}$. Subsequently, western blot analysis, MTT assay, immunostaining assay, PCR and immunofluorescence staining were performed as described below.

Western blot analysis. This was performed as previously described $(30,31)$. Total protein was prepared using extraction buffer comprising $\mathrm{NaCl} / \mathrm{P}_{\mathrm{i}}$ containing $0.5 \%$ Triton $\mathrm{X}-100$, $1 \mathrm{mM}$ EDTA, $1 \mathrm{mM}$ phenylmethyl sulfonyl fluoride, and complete protease inhibitors (Roche, Shanghai, China). The concentration of each protein lysate was determined using a BCA $^{\mathrm{TM}}$ protein assay kit (Thermo Fisher Scientific, Waltham, MA, USA). Equal amounts of total protein were subjected to $12 \% \mathrm{SDS} / \mathrm{PAGE}$. The samples were then transferred onto nitrocellulose membranes and blocked for $60 \mathrm{~min}$ at room temperature in $5 \%$ skim milk powder $(\mathrm{w} / \mathrm{v})$ in $\mathrm{NaCl} / \mathrm{P}_{\mathrm{i}}$ and protein was probed with antibodies against human YY1 (ab109228; 1:500) mouse double minute 2 homolog (ab38618; 1:500), ATPase copper transporting beta (ab124973, 1:500), integrina6 (ab235905, 1:500), signal transducer and activator of transcription 3 (ab68153, 1:500) or $\beta$-actin (ab8227, 1:500) (all from Abcam, Cambridge, MA, USA) and then with IRDyeTM-800 conjugated anti-rabbit secondary antibodies (1:10,000; ab150077; Abcam) all for $30 \mathrm{~min}$ at room temperature. The specific proteins were visualized using the Odyssey ${ }^{\mathrm{TM}}$ Infrared Imaging System (Gene Company, Lincoln, NE, USA).

MTT assay. To monitor the resistance to cisplatin, the U87MG, U87MG-CR and LN-229 cells were treated with $20 \mu \mathrm{M}$ cisplatin or DSMO for $24 \mathrm{~h}$. MTT assay was performed as previously described (32). Data were analyzed using software origin 7.5 (OriginLab, Northampton, MA, USA) to fit the sigmodial curve.

Sphere formation assay. The cells $\left(10^{3} / \mathrm{ml}\right)$ in serum-free RPMI-1640/1 mM Na-pyruvate were seeded on $0.5 \%$ agar pre-coated 6-well plates. After 1 week, half the medium was exchanged every 3rd day. Single spheres were selected and counted by an inverted microscope (TE2000-E2, Nikon Corporation, Tokyo, Japan).

Immunostaining assay for YY1 and CD133 in glioblastoma spheres. Single cell suspensions of glioblastoma cells transfected as indicated above were prepared and plated using ultra low adherent wells of 6-well plate at 5,000 cells/well in sphere formation medium (serum-free RPMI-1640/1 mM Na-pyruvate; Invitrogen), as described above. Following 7 days of treatment, the spheres were collected by centrifugation $\left(10,00 \mathrm{x} \mathrm{g}, 10 \mathrm{~min}, 4^{\circ} \mathrm{C}\right)$, washed with $1 \mathrm{X}$ PBS, and fixed with 3.7\% parformaldehyde for immunofluorescence staining. Anti-YY1 (ab109228; 1:500; Abcamand anti-CD133 antibodies (ab19898, 1:500) were used for immunostaining assay following the manufacturer's instructions and as previously described $(33,34)$. The coverslips were counterstained with 4'6-diamidino-2-phenylindole (DAPI; Thermo Fisher Scientific) for visualization of the nuclei. Microscopic analysis was performed with a confocal laser-scanning microscope (Leica Microsystems, Bensheim, Germany). The fluorescence intensities were measured in a few viewing areas for 300 cells per coverslip and analyzed using Image J $1.37 \mathrm{v}$ software (http://rsb.info.nih.gov/ij/index.html).

Real-time PCR for miRNA expression. Total RNA was isolated from the cells using the mirVana miRNA Isolation kit (Ambion, Austin, TX, USA). The detection of the mature form of miRNAs was performed using the mirVana qRT-PCR miRNA Detection kit and qRT-PCR Primer Sets, according to the manufacturer's instructions (Ambion). For the quantification PCR of miR-186, the forward primer was as follows: 5'-GCGGCGCAAAGAATTCTCCT-3', and the reverse primer was as follows: 5'-GTGCAGGGTCCGAGGT-3'. The quantification of PCR performed was performed using the $\Delta \Delta \mathrm{Cq}$ method (35). The U6 small nuclear RNA was used as an internal control.

Immunofluorescence staining. This was performed as previously described (36). Following transfection, the cells were fixed in $4 \%$ paraformaldehyde for $15 \mathrm{~min}$, and then blocked with goat serum blocking solution for $20 \mathrm{~min}$ at room temperature. Subsequently, rabbit antibody against YY1 (ab109228; 1:500; Abcam) were added, and the mixtures were incubated in a humid chamber overnight. After washing 3 times with $\mathrm{NaCl} / \mathrm{Pi}$, the cells were incubated with appropriate secondary antibodies (1:10,000; ab150077; Abcam) for $30 \mathrm{~min}$ at $37^{\circ} \mathrm{C}$. After washing with $\mathrm{NaCl} / \mathrm{Pi}$, the samples were observed under a laser scanning confocal microscope (Olympus, Tokyo, Japan). DAPI staining (blue) was used to highlight the nuclei. 
from the cells using TRIzol reagent (Invitrogen/Thermo Fisher Scientific). cDNA was synthesized from $1 \mu \mathrm{g}$ of total RNA in a $20 \mu \mathrm{l}$ reverse transcription (RT) system followed by PCR amplification in a $50 \mu \mathrm{l}$ PCR system performed using an RT-PCR kit (Cat no. A3500, Promega, Madison, WI, USA). The housekeeping gene, glyceraldehyde-3-phosphate dehydrogenase (GAPDH), was used as the RNA loading control. The PCR primer sequences were as follows: YY1 forward, 5'-CAG AAGCAGGTGCAGATCAAG-3' and reverse, 5'-GACCAC ATGGTGACCGAGAAC-3'; and GAPDH forward, 5'-ATT CAACGGCACAGTCAAGG-3' and reverse, 5'-GCAGAA GGGGCGGAGATGA-3'. PCR was conducted according to the manufacturer's instructions: The thermal cycle profile was as follows: Denaturation for $30 \mathrm{sec}$ at $95{ }^{\circ} \mathrm{C}$, annealing for $45 \mathrm{sec}$ at $52-58^{\circ} \mathrm{C}$ depending on the primers used, and extension for $45 \mathrm{sec}$ at $72^{\circ} \mathrm{C}$. Each PCR reaction was performed for 28-32 cycles. The PCR products were analyzed by agarose gel electrophoresis. Gels were photographed and densities of the bands were determined with a computerized image analysis system (Alpha Innotech, San Leandro, CA, USA). The area of each band was calculated as the integrated density value (IDV). qPCR for YY1 was performed using Power SYBR-Green PCR Master Mix (Applied Biosystems, Carlsbad, CA, USA) according to the manufacturer's instructions. Quantification of PCR performed was performed using the $\Delta \Delta$ Cq method (35).

Methods of bioinformatics. The analysis of potential miRNA target sites was carried out using the commonly used prediction algorithm, miRDB (http://mirdb.org/).

Northern blot analysis. Northern blot analysis of miRNAs, was performed as previously described (37). Probes were labeled with $\left[\gamma^{32} \mathrm{P}\right]$ ATP complementary to miR-186 and U6 snRNA.

Statistical analysis. Data are presented as the means \pm SEM. The Student's t-test (two-tailed) was used for comparisons between 2 groups. A value of $\mathrm{P}<0.05$ was considered to indicate a statistically significant difference.

\section{Results}

YY1 expression is increased in U87MG-CR cells and the silencing of YY1 sensitizes the U87MG-CR to cisplatin. In order to determine whether cisplatin resistance is associated with YY1 expression, we examined the YY1 protein concentrations in the U87MG and U87MG-CR cells. We observed that YY1 protein expression was increased in the U87MG-CR cells (Fig. 1A). To identify the role of YY1, we examined whether transfection with shYY1 plasmid would downregulate YY1 protein expression in the U87MG-CR cells. The results revealed that YY1 protein expression was inhibited by transfection with the shYY1 plasmid (Fig. 1B). To further determine whether YY1 affects the sensitivity of glioblastoma cells to cisplatin, we transfected the U87MG-CR cells with shYY1 plasmid or the scramble control and then performed MTT assay. We found that the silencing of YY1 transformed the U87MG-CR to cells to cisplatin-sensitive cells (U87MG cells), as evidenced by the decreased viability of the shYY1-transfected cells (Fig. 1C). We then examined
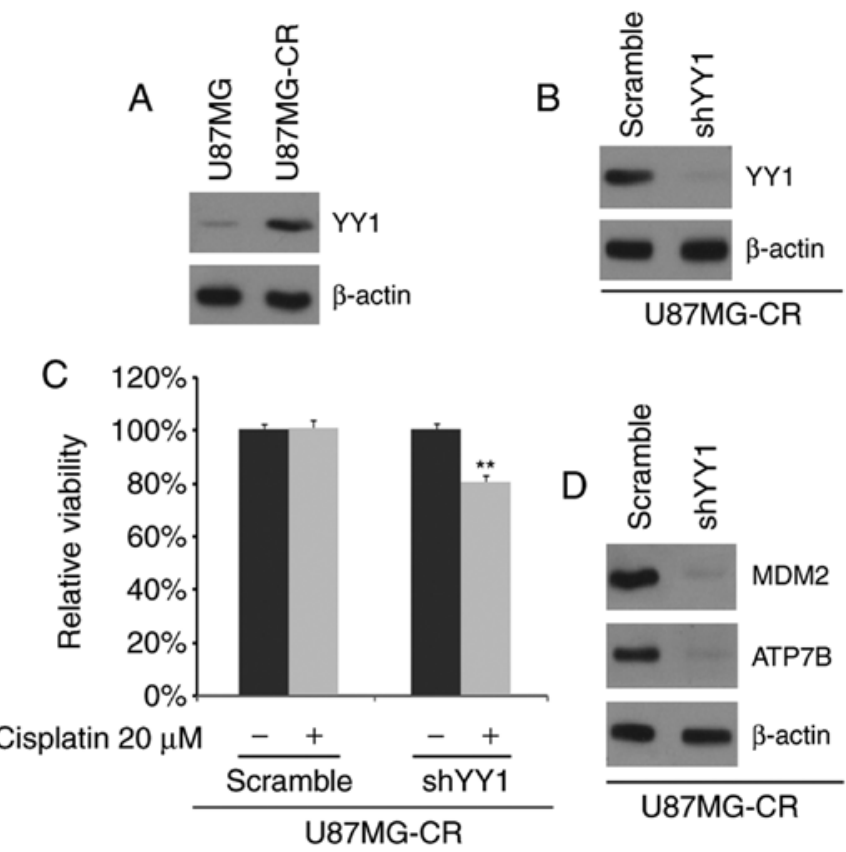

Figure 1. YY1 expression is increased in cisplatin-resistant U87MG cells (U87MG-CR cells) and its silencing sensitizes U87MG-CR cells to cisplatin. (A) Western blot analysis of YY1 expression in U87MG and U87MG-CR cells. (B) Western blot analysis of YY1 expression in U87MG-CR cells transfected with shYY1 plasmids or the scramble control. (C) MTT assay for the viability of the U87MG-CR cells. Cells transfected with the shYY1 plasmid or the scramble control were untreated or treated with cisplatin. (D) Western blot analysis of MDM2 and ATP7B expression in U87MG-CR cells transfected with shYY1 plasmid or the and scramble control. $\beta$-actin was used as a loading control; $\mathrm{n}=3$. $^{* *} \mathrm{P}<0.05$, compared with the shYY1-transfected cells not treated with cisplatin. YY1, Yin Yang 1; MDM2, mouse double minute 2 homolog; ATP7B, ATPase copper transporting beta.

the expression of MDM2 and ATP7B as MDM2 protein can confer the resistance of a human glioblastoma cell line to cisplatin-induced apoptosis and ATP7B is associated with cisplatin resistance $(38,39)$. In this study, we observed that the MDM2 and ATP7B protein expression levels were decreased in the U87MG-CR cells following transfection with shYY1 (Fig. 1D).

Overexpression of $Y Y 1$ promotes the resistance of $L N-229$ cells to cisplatin. To examine the effects of YY1, we examined whether YY1 protein expression was increased by YY1-expressing plasmids in LN-229 cells (cisplatin-sensitive cells). We observed that YY1 protein expression was increased following transfection with YY1-expressing plasmids (Fig. 2A). To identify whether the responses to cisplatin can be altered by YY1, we transfected the LN-229 cells with YY1-expressing plasmids and we then performed MTT assay. We found that the overexpression of YY1 promoted the resistance of LN-229 cells to cisplatin, as no marked difference in cell viability was observed between the cisplatin-treated or untreated LN-229-expressing cells (Fig. 2B).

Silencing of YY1 inhibits the formation of the GIC phenotype in U87MG-CR cells. To determine whether the silencing of YY1 affects the GIC phenotype of the U87MG-CR cells, we performed a sphere formation assay to assess the formation of GICs in the U87MG-CR cells. We observed that the cells 
A

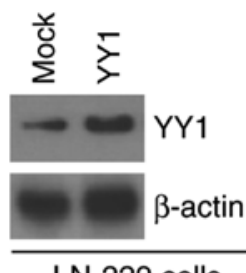

LN-229 cells
$\mathrm{B}$

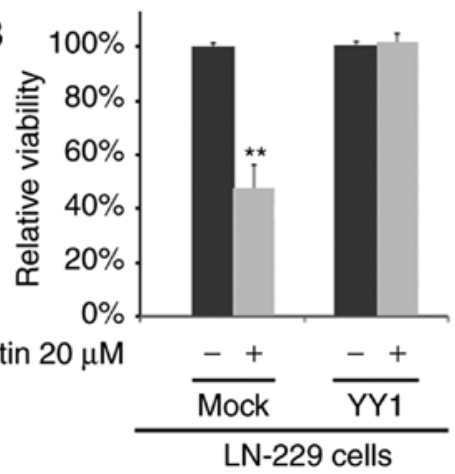

Figure 2. Overexpression of YY1 promotes the resistance of LN-229 cells to cisplatin. (A) Western blot analysis of YY1 in the LN-229 cells transfected with the YY1-expressing plasmid or the empty vector (mock). (B) MTT assay for the viability of LN-229 cells. Cells transfected with the YY1-expressing plasmid or the empty vector (mock) were untreated or treated with cisplatin. ${ }^{* *} \mathrm{P}<0.05$, compared with the mock-transfected cells not treated with cisplatin. YY1, Yin Yang 1.

transfected with shYY1 formed much smaller spheres after 14 days of culture as compared with the control cells (Fig. 3A). As CD133 expression is associated with the GIC phenotype in glioblastoma (40), in this study, we examined whether YY1 regulates CD133 protein expression. We performed immunostaining assay in the spheres isolated from the U87MG-CR cells transfected with the shYY1 plasmid or the scramble control. The results revealed that CD133 protein expression was decreased in the spheres isolated from the U87MG-CR cells transfected with the shYY1 plasmid (Fig. 3B). In addition, as integrin $\alpha 6$ regulates GICs and targeting integrin $\alpha 6$ in GICs inhibits self-renewal, proliferation and tumor formation capacity (41); thus, in this study, we also examined the expression of integrin $\alpha 6$. Moreover, as STAT3 is required for the proliferation and maintenance of multi-potency in glioblastoma stem cells (42), we also examined its expression. We found that the silencing of YY1 downregulated integrin $\alpha 6$ and STAT3 protein expression in the U87MG-CR cells (Fig. 3C).

miR-186 inhibits YY1 protein expression in U87MG-CR cells. To confirm whether YY1 is regulated by miRNAs, we used a commonly used prediction algorithm, miRDB (http://mirdb. org/) to analyze the 3'UTR of YY1. A total of 38 miRNAs were found by the algorithm. However, we were interested in miR-186, as miR-186 is a tumor suppressor gene by inhibiting oncogene expression $(24,26,43)$. Moreover, miR-186 may sensitize cancer cells to paclitaxel and cisplatin (25). The target sites on the 3'UTR of YY1 are shown in Fig. 4A. In an attempt to identify the role of miR-186 in regulating YY1 expression in glioblastoma, we transfected the U87MG-CR cells with pre-miR-186 and control miR. Following transfection, miR-186 expression was detected by real-time PCR and the results revealed that miR-186 expression was increased by transfection with pre-miR-186 (Fig. 4B). We then performed western blot analysis to detect YY1 protein expression in the U87MG-CR cells transfected with pre-miR-186 or control miR. We found that YY1 protein expression was inhibited by miR-186 (Fig. 4C). We then performed immunofluorescence analyses of the U87MG-CR cells transfected with pre-miR-186 anord control miR. We observed that YY1

A
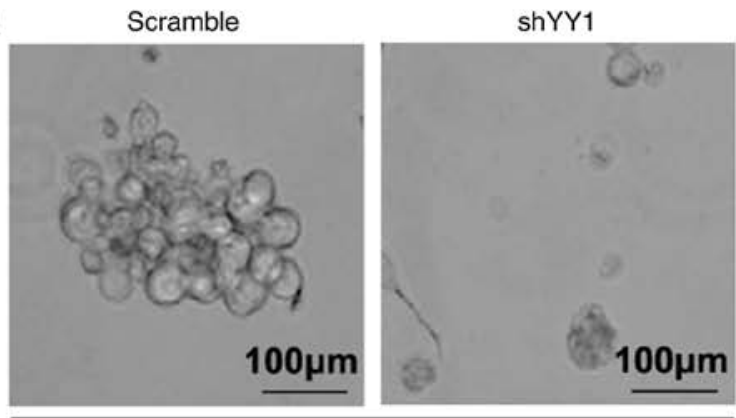

U87MG-CR

B
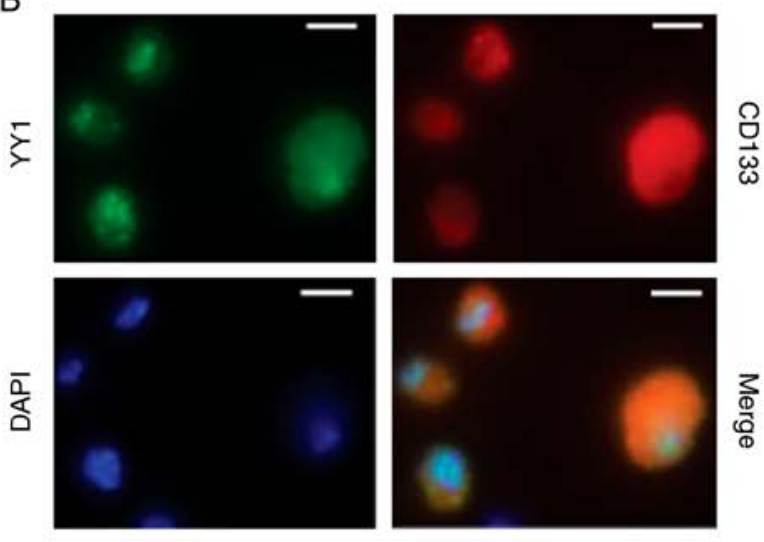

Scramble
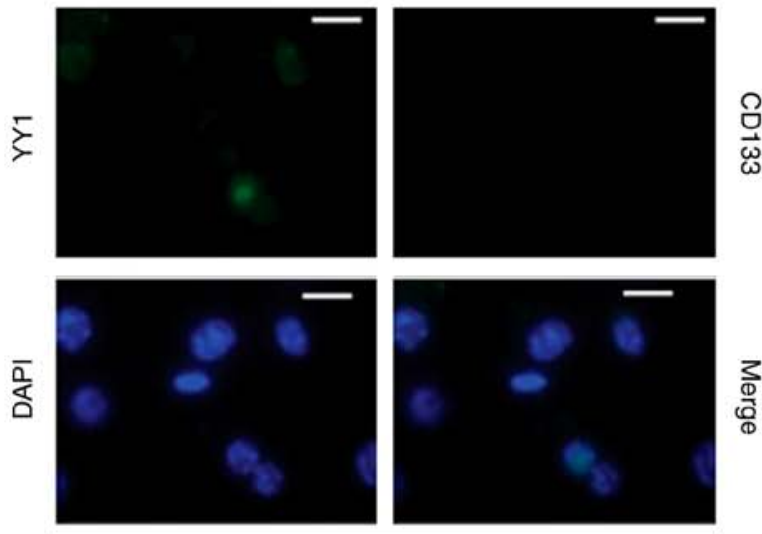

shYY1

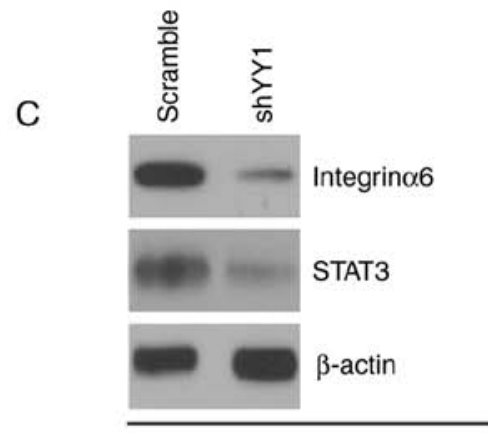

U87MG-CR

Figure 3. Silencing of YY1 inhibits the formation of the GIC phenotype in U87MG-CR cells. (A) Sphere growth for U87MG-CR cells transfected with the shYY1 plasmid of the scramble control. (B) Representative images of YY1 and CD133 immunofluorescence staining of spheres isolated from U87MG-CR cells transfected as indicated. Nuclei were counterstained with DAPI. Green indicates YY1 staining, red indicates CD133 fluorescence, and blue indicates DAPI (scale bars, $10 \mu \mathrm{m}$ ). (C) Western blot analysis of integrin $\alpha 6$ and STAT3 in U87MG-CR cells transfected with the shYY1 plasmid or the scramble control. $\beta$-actin was a loading control; $n=3$. YY1, Yin Yang 1; STAT3, signal transducer and activator of transcription 3. 


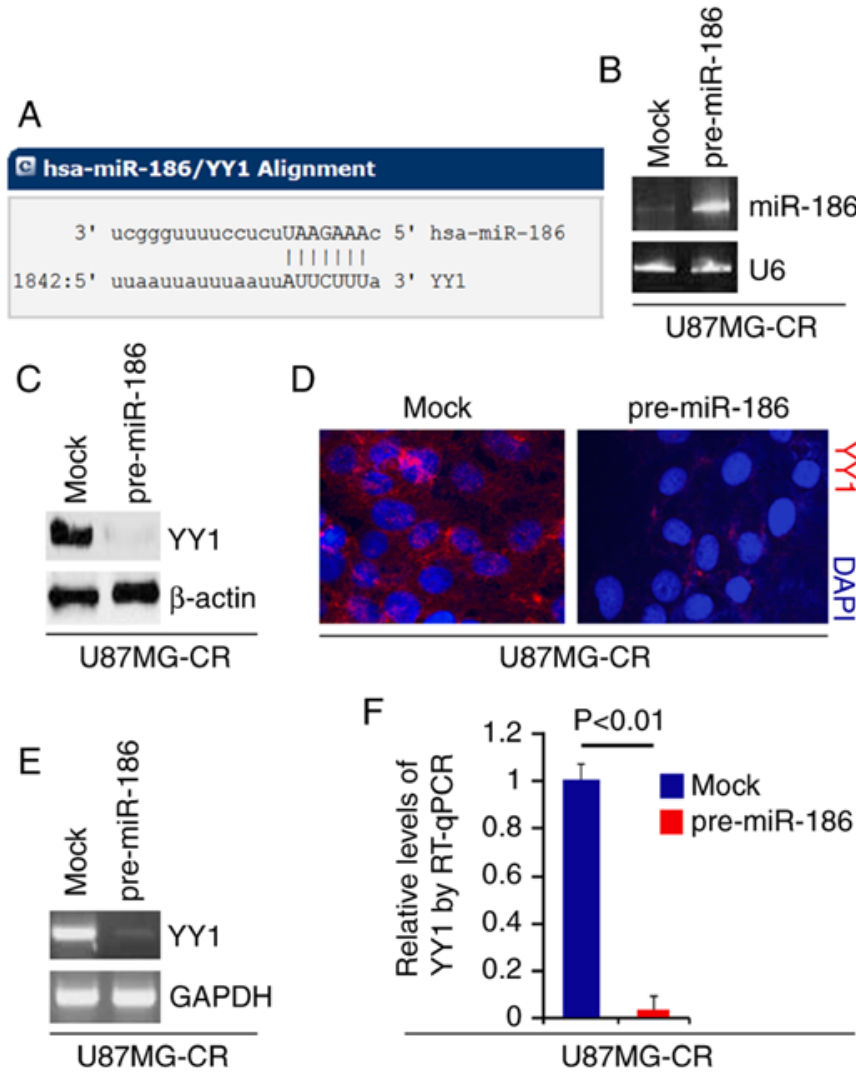

Figure 4. miR-186 inhibits YY1 expression in U87MG-CR cells. (A) Diagram demonstrating that $\mathrm{YY} 1$ is a target gene of miR-186, as predicted by miRanda. (B) Real-time PCR for miR-186 in U87MG-CR cells transfected with pre-miR-186 or the control miR (mock). U6 was used as a loading control. (C) Western blot analysis of YY1 expression in U87MG-CR cells transfected with pre-miR-186 or the control miR (mock). $\beta$-actin was used as a loading control. (D) Immunofluorescence analyses of YY1 in U87MG-CR cells transfected with pre-miR-186 or the control miR (mock). (E) RT-qPCR of YY1 in U87MG-CR cells transfected with pre-miR-186 or the control miR (mock). GAPDH was used as a loading control. (F) RT-qPCR of YY1 in U87MG-CR cells transfected with pre-miR-186 or the control miR (mock). GAPDH was used as a loading control; $n=3$. YY1, Yin Yang 1 .

protein expression was inhibited in the cells transfected with pre-miR-186 (Fig. 4D). To examine whether miR-186 degrades YY1 mRNA, we performed RT-qPCR and real-time PCR and we found that the overexpression of miR-186 degraded YY1 mRNA expresoin (Fig. 4E and F).

miR-186 expression is decreased in U87MG-CR cells and its overexpression reverses cisplatin resistance. To determine whether cisplatin resistance is associated with miR-186 expression, we performed northern blot analysis to detect miR-186 expression in U87MG cells and U87MG-CR cells. We observed that miR-186 expression was markedly decreased in the U87MG-CR cells (Fig. 5A). To further identify whether miR-186 can affect the resistance/sensitivity of U87MG-CR cells to cisplatin, we transfected the U87MG-CR cells with pre-miR-186 or control miR. We then performed MTT assay with the U87MG-CR cells treated as indicated (Fig. 5B). We found that the overexpression of miR-186 reversed cisplatin resistance, evidenced by the decreased viability of the U87MG-CR cells treated with cisplatin and transfected with pre-miR-186 (Fig. 5B). We also performed western blot

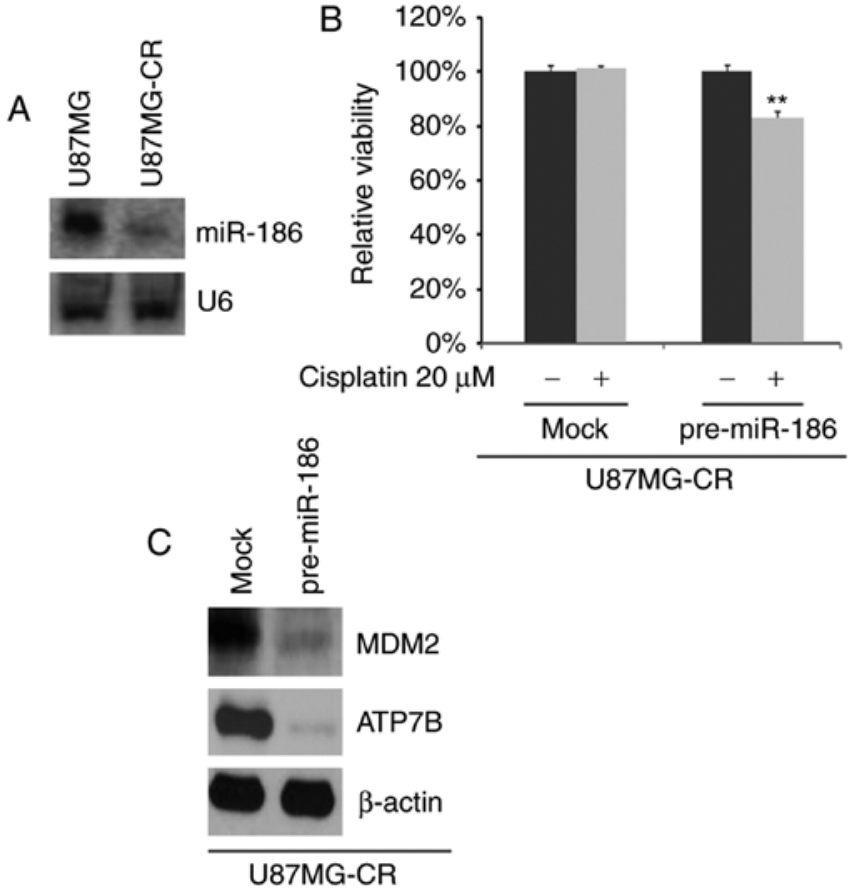

Figure 5. miR-186 is downregulated in U87MG-CR cells and its overexpression reverses cisplatin resistance. (A) Northern blot for miR-186 in U87MG and U87MG-res cells. U6 was used as a loading control. (B) MTT assay of the viability of U87MG-CR cells. Cells transfected with pre-miR-186 or the control miR (mock) were untreated or treated with cisplatin. (C) Western blot analysis of MDM2 and ATP7B in U87MG-CR cells transfected with pre-miR-186 or the control miR (mock). $\beta$-actin was used as a loading control; $n=3$. ${ }^{* *} \mathrm{P}<0.05$, compared with the pre-miR-186-transfected cells not treated with cisplatin. YY1, Yin Yang 1; MDM2, mouse double minute 2 homolog; ATP7B, ATPase copper transporting beta.

analysis to examine MDM2 and ATP7B protein expression in the U87MG-CR cells transfected with pre-miR-186 or control miR. The results revealed that MDM2 and ATP7B protein expression was inhibited by miR-186 (Fig. 5C).

miR-186 inhibits the formation of the GIC phenotype of U87MG-CR cells. To identify whether miR-186 can affect the GIC phenotype of U87MG-CR cells, we performed a sphere formation assay to assess the formation of GICs in the U87MG-CR cells. Sphere formation assay revealed that the overexpression of miR-186 inhibited the formation of GICs in U87MG-CR cells (Fig. 6A). Subsequently, to determine whether miR-186 regulates integrina6 and STAT3 protein expression, we performed western blot analysis of the U87MG-CR cells transfected with pre-miR-186 or control miR. We observed that integerin $\alpha 6$ and stat 3 protein expression levels were inhibited by miR-186 (Fig. 6B).

Silencing of miR-186 promotes the resistance of $L N-229$ cells to cisplatin. To determine whether miR-186 affects the sensitivity of the LN-229 cells to cisplatin, we transfected the LN-229 cells with anti-miR-186. We then performed real-time PCR to detect miR-186 expression in the LN-229 cells transfected with anti-miR-186 and scramble (mock). We observed that miR-186 expression was evidently decreased in the LN-229 cells transfected with anti-miR-186 (Fig. 7A). We then performed MTT assay of the LN-229 cells treated as indicated (Fig. 7B). The 
A

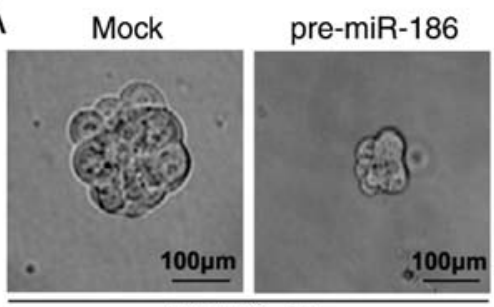

U87MG-CR

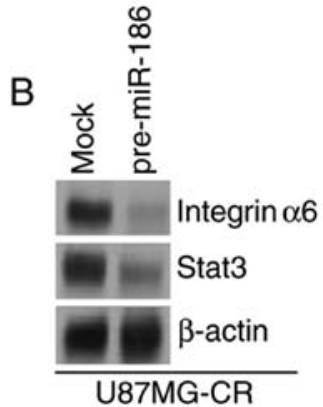

U87MG-CR
Figure 6. miR-186 inhibits formation of GICs phenotype in U87MG-CR cells. (A) Sphere growth of the U87MG-CR cells transfected with pre-miR-186 or the control miR (mock). (B) Western blot analysis of integrina6 and STAT3 expression in U87MG cells transfected with pre-miR-186 or the control miR (mock). $\beta$-actin was used as a loading control; $n=3$. YY1, Yin Yang 1; STAT3, signal transducer and activator of transcription 3 .

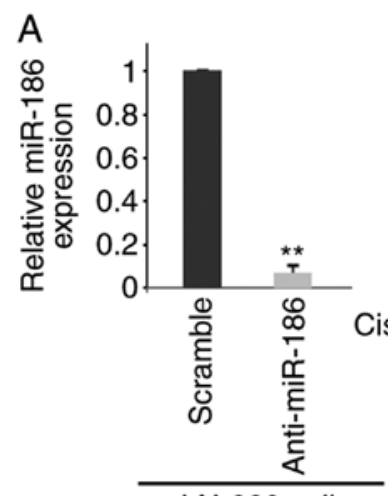

B

LN-229 cells

Figure 7. Silencing of miR-186 promotes the resistance of $\mathrm{LN}-229$ cells to cisplatin. (A) Real-time PCR of miR-186 expression in LN-229 cells transfected with anti-miR-186 or the scramble control. GAPDH was used as a loading control. (B) MTT assay of the viability of the LN-229 cells. Cells transfected with anti-miR-186 or the scramble control were untreated or treated with cisplatin; $n=3$. ${ }^{* *} \mathrm{P}<0.05$, compared with the anti-miR-186-transfected or mock-transfected (scramble) cells not treated with cisplatin.

results revealed that the overexpression of miR-186 promoted cisplatin resistance, as the cells transfected with anti-miR-186 and treated with cisplatin exhibited no marked difference in viability compared with the anti-miR-186-transfected cells not treated with cisplatin (Fig. 7B).

\section{Discussion}

Cisplatin is a neutral, square planar platinum (II) complex containing two chloride ligands oriented in a cis configuration. It has become one of the most effective chemotherapeutic agents for the treatment of glioblastoma (44). However, intrinsic or acquired resistance to cisplatin reduces its efficacy (45). The mechanisms of resistance include miRNA deregulation and the formation of GICs (46-48). miR-186 has been demonstrated to play a significant role as a tumor suppressor in many types of cancer (24-26). Nevertheless, its biological function in glioblastoma remains unknown. In the current study, we found that miR-186 played an important role in the formation of GICs and in the regulation of cisplatin resistance. These findings provide novel insight into the potential roles of miR-186 in promoting the formation of GICs and conferring chemoresistance in glioblastoma. MDM2 protein can confer the resistance of a human glioblastoma cell line to cisplatin (39). We demonstrated that the overexpression of miR-186 inhibited MDM2 protein expression. The ATP7B product, a protein of 1465 amino acids (ATP7B), is expressed pre-dominantly in the liver, kidneys and placenta in humans (49). ATP7B expression is associated with cisplatin resistance (38). In this study, we found that ATP7B expression was inhibited by miR-186 in U87MG-CR cells.

A number of studies have relied on the enrichment of GICs based on the expression of the cell surface protein CD133 (prominin-1) (50,51), which has also been used as a selection marker for neural stem cells (51). In this study, we demonstrated that miR-186 inhibited CD133 expression. Moreover, integrina6 is co-expressed with conventional GIC markers (41); STAT3 is required for maintenance of multipotency in GICs (42). Herein, we observed that the overexpression of miR-186 significantly inhibited integrin $\alpha 6$ and STAT3 protein expression in the U87MG-CR cells.

YY1 plays an important role in the EGFR-Src-p38 signaling cascade in glioblastoma. However, its roles and regulatory mechanisms have not yet been fully elucidated. EGFR signaling plays an important role in drug resistance for the treatment of glioblastoma (52) and EGFR inhibitor can enhance cisplatin sensitivity of human glioma cells (52). We demonstrated herein that YY1 expression was increased in cisplatin-resistant U87MG cells and that the silencing of YY1 sensitized the U87MG-CR cells to cisplatin. In addition, we observed that YY1 expression was regulated by miR-186 in U87MG-CR cells.

Recently, the U-87 MG cell line from ATCC was reported to be contaminated or misidentified (28). It has been proposed as a glioblastoma cell line whose origin is unknown (28). However, the U-87 MG cell line is still widely used for glioblastoma research (25). In the present study, the U87MG and LN-229 cells were used. The results were same from the 2 cell lines. Thus, the contamination or misidentification may not affect the conclusions presented herein.

In conclusion, elucidating the mechanisms through which miR-186 reverses cisplatin resistance and inhibits the formation of the GIC phenotype by degrading YY1 in glioblastoma may enhance our understanding of the molecular mechanisms of cisplatin resistance in glioblastoma. As shown by our findings, the restoration of miR-186 expression may represent a promising therapeutic strategy with which to inhibit YY1-mediated cisplatin resistance. However, the roles of miR-186 and YY1 require further confirmation by in vivo studies.

\section{Acknowledgements}

Not applicable.

\section{Funding}

The present study was supported by Linyi People's Hospital.

\section{Availability of data and materials}

The datasets used and/or analyzed during the current study are available from the corresponding author on reasonable request. 


\section{Authors' contributions}

JL and FG conceived the study, collected the experimental data and wrote a draft of the manuscript. JS contributed to the experimental work and data analysis. All authors edited and approved the final version of the manuscript.

\section{Ethics approval and consent to participate}

Not applicable.

\section{Patient consent for publication}

Not applicable.

\section{Competing interests}

The authors declare that they have no competing interests.

\section{References}

1. Buckner JC: Factors influencing survival in high-grade gliomas Semin Oncol 30: 10-14, 2003.

2. Curran WJ Jr, Scott CB, Horton J, Nelson JS, Weinstein AS, Fischbach AJ, Chang CH, Rotman M, Asbell SO, Krisch RE, et al: Recursive partitioning analysis of prognostic factors in three Radiation Therapy Oncology Group malignant glioma trials J Natl Cancer Inst 85: 704-710, 1993.

3. DeAngelis LM: Brain tumors. N Eng J Med 344: 114-123, 2001.

4. Ohgaki $\mathrm{H}$ and Kleihues P: Epidemiology and etiology of gliomas. Acta Neuropathol 109: 93-108, 2005.

5. Bao S, Wu Q, McLendon RE, Hao Y, Shi Q, Hjelmeland AB Dewhirst MW, Bigner DD and Rich JN: Glioma stem cells promote radioresistance by preferential activation of the DNA damage response. Nature 444: 756-760, 2006.

6. Bao S, Wu Q, Sathornsumetee S, Hao Y, Li Z, Hjelmeland AB Shi Q, McLendon RE, Bigner DD and Rich JN: Stem cell-like glioma cells promote tumor angiogenesis through vascular endothelial growth factor. Cancer Res 66: 7843-7848, 2006.

7. Zeppernick F, Ahmadi R, Campos B, Dictus C, Helmke BM, Becker N, Lichter P, Unterberg A, Radlwimmer B and Herold-Mende CC: Stem cell marker CD133 affects clinical outcome in glioma patients. Clin Cancer Res 14: 123-129, 2008.

8. Shi Y, Seto E, Chang LS and Shenk T: Transcriptional repression by YY1, a human GLI-Krüippel-related protein, and relief of repression by adenovirus E1A protein. Cell 67: 377-388, 1991.

9. Park Kand Atchison ML:Isolation of acandidate repressor/activator, NF-E1 (YY-1, delta), that binds to the immunoglobulin kappa 3 'enhancer and the immunoglobulin heavy-chain mu E1 site. Proc Natl Acad Sci USA 88: 9804-9808, 1991.

10. Hariharan N, Kelley DE and Perry RP: Delta, a transcription factor that binds to downstream elements in several polymerase II promoters, is a functionally versatile zinc finger protein. Proc Natl Acad Sci USA 88: 9799-9803, 1991.

11. Flanagan JR, Becker KG, Ennist DL, Gleason SL, Driggers PH, Levi BZ, Appella E and Ozato K: Cloning of a negative transcription factor that binds to the upstream conserved region of Moloney murine leukemia virus. Mol Cell Biol 12: 38-44, 1992.

12. Thomas MJ and Seto E: Unlocking the mechanisms of transcription factor YY1: Are chromatin modifying enzymes the key? Gene 236: 197-208, 1999.

13. Shi Y, Lee JS and Galvin KM: Everything you have ever wanted to know about Yin Yang 1...... Biochim Biophys Acta 1332: F49-F66, 1997.

14. Iuchi S and Kuldell N: Zinc Finger Proteins: From Atomic Contact to Cellular Function. Springer Science Business Media, New York, NY, 2007.

15. Gordon S, Akopyan G, Garban H and Bonavida B: Transcription factor YY1: Structure, function, and therapeutic implications in cancer biology. Oncogene 25: 1125-1142, 2006.

16. Wilkinson FH, Park K and Atchison ML: Polycomb recruitment to DNA in vivo by the YY1 REPO domain. Proc Natl Acad Sci USA 103: 19296-19301, 2006.
17. Thomassen M, Tan Q and Kruse TA: Gene expression meta-analysis identifies metastatic pathways and transcription factors in breast cancer. BMC Cancer 8: 394, 2008

18. Wan M, Huang W, Kute TE, Miller LD, Zhang Q, Hatcher H, Wang J, Stovall DB, Russell GB, Cao PD, et al: Yin Yang 1 plays an essential role in breast cancer and negatively regulates $\mathrm{p} 27$. Am J Pathol 180: 2120-2133, 2012.

19. Chinnappan D, Xiao D, Ratnasari A, Andry C, King TC and Weber HC: Transcription factor YY1 expression in human gastrointestinal cancer cells. Int J Oncol 34: 1417-1423, 2009.

20. Kang W, Tong J, Chan A, Zhao J, Dong Y, Wang S, Yang W, Sin FM, Ng SS, Yu J, et al: Yin Yang 1 contributes to gastric carcinogenesis and its nuclear expression correlates with shorter survival in patients with early stage gastric adenocarcinoma. J Transl Med 12: 80, 2014

21. Seligson D, Horvath S, Huerta-Yepez S, Hanna S, Garban H, Roberts A, Shi T, Liu X, Chia D, Goodglick L and Bonavida B: Expression of transcription factor Yin Yang 1 in prostate cancer. Int J Oncol 27: 131-141, 2005.

22. Sarkar FH, Li Y, Wang Z, Kong D and Ali S: Implication of microRNAs in drug resistance for designing novel cancer therapy. Drug Resist Updat 13: 57-66, 2010.

23. Gal H, Pandi G, Kanner AA, Ram Z, Lithwick-Yanai G, Amariglio N, Rechavi G and Givol D: MIR-451 and Imatinib mesylate inhibit tumor growth of Glioblastoma stem cells. Biochem Biophys Res Commun 376: 86-90, 2008.

24. Ruan T, He X, Yu J and Hang Z: MicroRNA-186 targets Yes-associated protein 1 to inhibit Hippo signaling and tumorigenesis in hepatocellular carcinoma. Oncol Lett 11: 2941-2945, 2016.

25. Sun KX, Jiao JW, Chen S, Liu BL and Zhao Y: MicroRNA-186 induces sensitivity of ovarian cancer cells to paclitaxel and cisplatin by targeting ABCB1. J Ovarian Res 8: 80, 2015.

26. Zhang TJ, Wang YX, Yang DQ, Yao DM, Yang L, Zhou JD, Deng ZQ, Wen XM, Guo H, Ma JC, et al: Down-regulation of miR-186 correlates with poor survival in de novo acute myeloid leukemia. Clin Lab 62: 113-120, 2015.

27. Wang $\mathrm{F}$, Jiang $\mathrm{H}$, Wang $\mathrm{S}$ and Chen B: Dual functional MicroRNA-186-5p targets both FGF2 and RelA to suppress tumorigenesis of glioblastoma multiforme. Cell Mol Neurobiol 37: 1433-1442, 2017.

28. Allen M, Bjerke M, Edlund H, Nelander S and Westermark B Origin of the U87MG glioma cell line: Good news and bad news. Sci Transl Med 8: 354re3, 2016.

29. Sun L, Yao Y, Liu B, Lin Z, Lin L, Yang M, Zhang W, Chen W, Pan C, Liu Q, et al: miR-200b and miR-15b regulate chemotherapy-induced epithelial-mesenchymal transition in human tongue cancer cells by targeting BMI1. Oncogene 31: 432-445, 2012.

30. Liao XH,Lu DL, Wang N,Liu LY, Wang Y,Li YQ, Yan TB,SunXG, Hu P and Zhang TC: Estrogen receptor $\alpha$ mediates proliferation of breast cancer MCF-7 cells via a p21/PCNA/E2F1-dependent pathway. FEBS J 281: 927-942, 2014.

31. Xiang Y, Lu DL, Li JP, Yu CX, Zheng DL, Huang X, Wang ZY, $\mathrm{Hu}$ P, Liao XH and Zhang TC: Myocardin inhibits estrogen receptor alpha-mediated proliferation of human breast cancer MCF-7 cells via regulating MicroRNA expression. IUBMB Life 68: 477-487, 2016.

32. Kataoka J, Shiraha H, Horiguchi S, Sawahara H, Uchida D, Nagahara T, Iwamuro M, Morimoto H, Takeuchi Y, Kuwaki K, et al: Loss of Runt-related transcription factor 3 induces resistance to 5-fluorouracil and cisplatin in hepatocellular carcinoma. Oncol Rep 35: 2576-2582, 2016.

33. Ali S, Ahmad A, Banerjee S, Padhye S, Dominiak K, Schaffert JM, Wang Z, Philip PA and Sarkar FH: Gemcitabine sensitivity can be inducedinpancreaticcancercellsthroughmodulation ofmiR-200and miR-21 expression by curcumin or its analogue CDF. Cancer Res 70: 3606-3617, 2010.

34. Kong D, Li Y, Wang Z, Banerjee S, Ahmad A, Kim HR and Sarkar FH: miR-200 regulates PDGF-D-mediated epithelialmesenchymal transition, adhesion, and invasion of prostate cancer cells. Stem Cells 27: 1712-1721, 2009.

35. Livak KJ and Schmittgen TD: Analysis of relative gene expression data using real-time quantitative PCR and the 2(-Delta Delta C(T) method. Methods 25: 402-408, 2001.

36. Jia Y, Chen J, Zhu H, Jia ZH and Cui MH: Aberrantly elevated redox sensing factor $\mathrm{Nrf} 2$ promotes cancer stem cell survival via enhanced transcriptional regulation of ABCG2 and Bcl-2/Bmi-1 genes. Oncol Rep 34: 2296-2304, 2015. 
37. Yu J, Ryan DG, Getsios S, Oliveira-Fernandes M, Fatima A and Lavker RM: MicroRNA-184 antagonizes microRNA-205 to maintain SHIP2 levels in epithelia. Proc Natl Acad Sci USA 105: 19300-19305, 2008.

38. Komatsu M, Sumizawa T, Mutoh M, Chen ZS, Terada K Furukawa T, Yang XL, Gao H, Miura N, Sugiyama T and Akiyama S: Copper-transporting P-type adenosine triphosphatase (ATP7B) is associated with cisplatin resistance. Cancer Res 60: 1312-1316, 2000

39. Kondo S, Barnett GH, Hara H, Morimura T and Takeuchi J: MDM2 protein confers the resistance of a human glioblastoma cell line to cisplatin-induced apoptosis. Oncogene 10: 2001-2006, 1995.

40. Brescia P, Ortensi B, Fornasari L, Levi D, Broggi G and Pelicci G: CD133 is essential for glioblastoma stem cell maintenance. Stem Cells 31: 857-869, 2013.

41. Lathia JD, Gallagher J, Heddleston JM, Wang J, Eyler CE, Macswords J, Wu Q, Vasanji A, McLendon RE, Hjelmeland AB and Rich JN: Integrin alpha 6 regulates glioblastoma stem cells. Cell Stem Cell 6: 421-432, 2010.

42. Sherry MM, Reeves A, Wu JK and Cochran BH: STAT3 is required for proliferation and maintenance of multipotency in glioblastoma stem cells. Stem Cells 27: 2383-2392, 2009.

43. He W, Feng J, Zhang Y, Wang Y, Zang W and Zhao G: MicroRNA-186 inhibits cell proliferation and induces apoptosis in human esophageal squamous cell carcinoma by targeting SKP2. Lab Invest 96: 317-324, 2016.

44. Brandes AA, Basso U, Reni M, Vastola F, Tosoni A, Cavallo G, Scopece L, Ferreri AJ, Panucci MG, Monfardini S, et al: First-line chemotherapy with cisplatin plus fractionated temozolomide in recurrent glioblastoma multiforme: A phase II study of the Gruppo Italiano Cooperativo di Neuro-Oncologia. J Clin Oncol 22: 1598-1604, 2004.
45. Timmer-Bosscha H, Mulder NH and de Vries E: Modulation of cis-diamminedichloroplatinum(II) resistance: A review. Br J Cancer 66: 227-238, 1992.

46. Chen W, Yang Y, Chen B, Lu P, Zhan L, Yu Q, Cao K and Li Q: miR-136 targets E2F1 to reverse cisplatin chemosensitivity in glioma cells. J Neurooncol 120: 43-53, 2014.

47. Wang Q, Wang Z, Chu L, Li X, Kan P, Xin X, Zhu Y and Yang P: The effects and molecular mechanisms of miR-106a in multidrug resistance reversal in human glioma U87/DDP and U251/G cell lines. PLoS One 10: e0125473, 2015.

48. Eramo A, Ricci-Vitiani L, Zeuner A, Pallini R, Lotti F, Sette G, Pilozzi E, Larocca LM, Peschle C and De Maria R: Chemotherapy resistance of glioblastoma stem cells. Cell Death Differ 13: 1238-1241, 2006.

49. Terada K, Schilsky ML, Miura N and Sugiyama T: ATP7B (WND) protein. Int J Biochem Cell Biol 30: 1063-1067, 1998.

50. Bidlingmaier S, Zhu X and Liu B: The utility and limitations of glycosylated human CD133 epitopes in defining cancer stem cells. J Mol Med (Berl) 86: 1025-1032, 2008.

51. Uchida N, Buck DW, He D, Reitsma MJ, Masek M, Phan TV, Tsukamoto AS, Gage FH and Weissman IL: Direct isolation of human central nervous system stem cells. Proc Natl Acad Sci USA 97: 14720-14725, 2000.

52. Zhang Y, Xing X, Zhan H, Li Q, Fan Y, Zhan L, Yu Q and Chen J: EGFR inhibitor enhances cisplatin sensitivity of human glioma cells. J Huazhong Univ Sci Technol Med Sci 31: 773-778, 2011. 